\title{
The combination of temsirolimus and chloroquine increases radiosensitivity in colorectal cancer cells
}

\author{
HIROSHI SHIRATORI, KAZUSHIGE KAWAI, KEISUKE HATA, TOSHIAKI TANAKA, TAKESHI NISHIKAWA, \\ KENSUKE OTANI, KAZUHITO SASAKI, MANABU KANEKO, KOJI MURONO, \\ SHIGENOBU EMOTO, HIROFUMI SONODA and HIROAKI NOZAWA \\ Division of Surgical Oncology, Department of Surgery, Faculty of Medicine, \\ The University of Tokyo, Tokyo 113-8655, Japan
}

Received December 19, 2018; Accepted April 19, 2019

DOI: $10.3892 /$ or.2019.7134

\begin{abstract}
The PI3K/AKT/mTOR pathway and autophagy are known to play important roles in cancer radioresistance. The aim of the present study was to investigate whether the combination of temsirolimus (TEM), an mTOR inhibitor, and chloroquine (CQ), an autophagy inhibitor, can increase radiosensitivity in colorectal cancer (CRC) cells. The efficacies of TEM and/or CQ as radiosensitizers were examined using clonogenic assays in CRC cell lines SW480 and HT-29. The expression levels of the phosphorylated isoforms of S6 and 4E-BP1, downstream proteins of mTOR, as well as the expression levels of p62 and LC3, autophagy-related proteins, were assessed by western blot analysis. The formation of acidic organelles was detected in acridine orange-stained cells. Apoptosis and caspase activity were assessed using flow cytometry. The results revealed that ionizing radiation (IR) activated the downstream proteins of mTOR and induced autophagy. In the clonogenic assays, neither TEM nor CQ influenced the efficacy of IR, whereas their combination significantly increased the dose-dependent efficacy of IR. TEM inhibited phosphorylation of the downstream proteins of mTOR and induced autophagy. CQ inhibited autophagy in the late phase and did not influence the downstream proteins of mTOR. TEM and CQ inhibited both the phosphorylation of downstream proteins of mTOR and autophagy. Cell death analysis revealed that the combination of TEM and CQ strongly induced apoptosis in cells exposed to IR. In conclusion, the combination of TEM and CQ increased radiosensitivity in CRC cells through co-inhibition of mTOR and autophagy.
\end{abstract}

Correspondence to: Dr Hiroshi Shiratori, Division of Surgical Oncology, Department of Surgery, Faculty of Medicine, The University of Tokyo, 7-3-1 Hongo, Bunkyo-ku, Tokyo 113-8655, Japan E-mail: tori.shior@gmail.com

Key words: colorectal cancer, radiosensitivity, mTOR, autophagy, temsirolimus, chloroquine

\section{Introduction}

Preoperative chemoradiotherapy (CRT) is considered a standard therapy for local control in patients with locally advanced rectal cancer. Pathological complete response (pCR) in the patients treated with CRT implies the absence of residual cancer in the resected specimen. Since the recurrence rate after curative surgery in the patients with $\mathrm{pCR}$ has been reported to be lower than that in non-responders (1), increasing the pCR rate is necessary to improve patient prognosis. However, 5-FU-based conventional CRT has been revealed to result in pCR for only $13-17 \%$ of patients (2), and these results are insufficient. Hence, chemotherapeutic regimens for increasing tumor radiosensitivity are being investigated worldwide.

Preclinical studies on solid cancer cells revealed that ionizing radiation (IR) activated the PI3K/AKT/mTOR signaling pathway, which was considered to be one of the radioresistance mechanisms $(3,4)$. However, in a phase-II clinical trial for patients with locally advanced rectal cancer, CRT combined with rapamycin, an mTOR inhibitor, resulted in pCR in $3 \%$ of patients and the radiosensitizing effect of mTOR inhibitor was not observed (5).

An mTOR inhibitor is known to induce autophagy by dephosphorylating UNC-51-like kinase (ULK1). Autophagy is considered as a cause for chemo/radiotherapy resistance in cancer cells due to its function of self-protection and self-degradation $(6,7)$. This cytoprotective function of autophagy was reported to play a role in resistance to the antitumor effect of mTOR inhibitor (8). In addition, in a previous study, it was demonstrated that the combination of temsirolimus (TEM), an mTOR inhibitor, and chloroquine (CQ), an autophagy inhibitor, improved the antitumor effect in colorectal cancer (CRC) cells (9). Based on this result, we hypothesized that the simultaneous inhibition of mTOR signaling and autophagy is essential to enhance radiosensitivity. Therefore, in the present study, we aimed to determine whether the co-inhibition of mTOR and autophagy using TEM and CQ enhanced radiosensitivity in CRC cells.

\section{Materials and methods}

Cell culture and reagents. The human CRC cell lines, HT-29 and SW480, were purchased from the Japanese Cancer 
Research Resource Bank. Cells were cultured in RPMI-1640 medium (Sigma-Aldrich; Merck KGaA, Darmstadt, Germany) supplemented with $5 \%$ fetal bovine serum (FBS) and $1 \%$ antibiotic/antimycotic solution in a $5 \% \mathrm{CO}_{2}$ incubator at $37^{\circ} \mathrm{C}$. The mTOR inhibitor TEM and the autophagy inhibitor chloroquine diphosphate (CQ) were purchased from Sigma-Aldrich; Merck KGaA. Rabbit antibodies against phospho-S6 ribosomal protein (Ser235/236), S6 ribosomal protein (5G10), phospho-eukaryotic translation initiation factor 4E-binding protein 1 (phospho-4E-BP1; Thr37/46), and 4E-BP1 were purchased from Cell Signaling Technology (Tokyo, Japan); those against microtubule-associated protein light chain 3 beta (LC3B), protein 62 [p62; also known as sequestosome-1 (SQSTM1)], and $\beta$-actin were obtained from Medical and Biological Laboratories (MBL, Nagoya, Japan); and alkaline phosphatase-labeled goat anti-rabbit antibody was purchased from Abcam (Cambridge, UK).

Preparation of radiosensitizers and exposure to IR. The experimental doses of TEM and CQ were determined to be $80 \mathrm{nM}$ and $20 \mu \mathrm{M}$, respectively based on a previous study (9). The cells were pretreated with TEM (TEM group), CQ (CQ group), both (TEM+CQ group), or no treatment (the control group) for $1 \mathrm{~h}$ before exposure to IR. Cells were irradiated at doses of $\sim 6 \mathrm{~Gy}(1.0 \mathrm{~Gy} / \mathrm{sec})$ with an X-ray generator (Pantac HF350; Kyoto, Japan). Irradiated cells were used in subsequent experiments. The experimental radiation dose was determined according to several preceding studies (10-12), including our previous study using colorectal cell lines (13).

Clonogenic assay. The SW480 and HT-29 cells from each group were seeded at densities of 200-1,200 cells in a 6-well plate. Cells were pretreated with or without TEM and/or CQ for $1 \mathrm{~h}$ followed by exposure to IR at doses of $0,2,4$ or $6 \mathrm{~Gy}$. After incubation for $24 \mathrm{~h}$, the medium was changed to a fresh one without drugs. The medium was then changed to a fresh one every $48 \mathrm{~h}$ for 14 days. Then, the cells were fixed with $70 \%$ ethanol at room temperature for $30 \mathrm{~min}$, and stained with $0.5 \%$ crystal violet solution at room temperature for $30 \mathrm{~min}$, and the colonies were counted. The surviving fraction (SF) in each group was calculated as follows: $\mathrm{SF}=$ no. of colonies with IR/no. of colonies without IR. For each experimental condition, colonies from three different wells were counted. All SF values were expressed as the mean \pm standard deviation $(\mathrm{SD})$.

Cell proliferation assay. SW480 and HT-29 cells $\left(5 \times 10^{4}\right.$ cells/well) were seeded in 96-well flat-bottomed plates and incubated for $72 \mathrm{~h}$ with or without TEM and/or CQ after exposure to IR at a dose of $4 \mathrm{~Gy}$. Then, MTS reagent (Promega Corp., Madison, WI, USA) was added to each well, cells were incubated for $3 \mathrm{~h}$, and spectrophotometric assessment was performed with a 490-nm filter using Varioskan Flash, a microplate reader (Life Technologies; Thermo Fisher Scientific, Inc.). All experiments were performed in triplicate wells, and the data for cell proliferation were expressed as the mean $\pm \mathrm{SD}$.

Western blot analysis. SW480 and HT-29 cells were incubated for $24 \mathrm{~h}$ with or without TEM and/or CQ after exposure to IR at a dose of $4 \mathrm{~Gy}$. Whole cell protein extracts were obtained by lysing cells in Bolt LDS sample buffer (Life Technologies; Thermo Fisher Scientific, Inc., Waltham, MA, USA) supplemented with protease and phosphatase inhibitors (Roche Diagnostics, Basel, Switzerland). Solubilized proteins were quantified using the Qubit protein assay kit (Life Technologies; Thermo Fisher Scientific, Inc.) and $40 \mu \mathrm{g}$ of protein samples were separated by sodium dodecyl sulfate-polyacrylamide gel electrophoresis (SDS-PAGE) with 4-12\% Bis-Tris Gel (Life Technologies; Thermo Fisher Scientific, Inc.) and transferred to polyvinylidene difluoride (PVDF) membranes (pore size $0.2 \mu \mathrm{m}$ ) using the Bolt system (Life Technologies; Thermo Fisher Scientific, Inc.). After blocking with the iBind solution (Life Technologies; Thermo Fisher Scientific, Inc.) at room temperature for $5 \mathrm{~min}$, western blotting was performed using the iBind Western System (Life Technologies; Thermo Fisher Scientific, Inc.), according to the manufacturer's instructions; the membranes were incubated with rabbit antibodies against phospho-S6 ribosomal protein (Ser235/236; dilution 1:1,000; cat. no. 4857), S6 ribosomal protein (5G10; dilution 1:1,000; cat. no. 2217), phospho-4E-BP1 (Thr37/46; dilution 1:1,000; cat. no. 2855), 4E-BP1 (dilution 1:1,000; cat. no. 9452) all from CST; LC3B (dilution 1:1,000; cat. no. PM036), p62, also known as sequestosome-1 (SQSTM1) (dilution 1:1,000; cat. no. PM045), or $\beta$-actin (dilution 1:1,000; cat. no. PM053) all from MBL at room temperature for $3 \mathrm{~h}$, followed by alkaline phosphatase-labeled goat anti-rabbit antibody (dilution 1:2,000; cat. no. ab97048; Abcam) at room temperature for $2 \mathrm{~h}$. The membranes were incubated in CDP-Star solution (Life Technologies; Thermo Fisher Scientific, Inc.) for $5 \mathrm{~min}$, and chemiluminescence was detected using ChemiDoc XRS system (Bio-Rad Laboratories, Inc., Tokyo, Japan). The experiment was performed three times. The density of each band was measured using ImageJ software (version 1.4.3; National Institutes of Health, Bethesda, MD, USA) and expressed as the mean $\pm \mathrm{SD}$. The phosphorylated isoform fraction of S6 and 4E-BP1 were normalized by the respective total protein levels.

Acidic vesicular organelle (autophagosome) detection. SW480 and HT-29 cells were prepared and treated as aforementioned. The cells were collected in FACS tubes and stained with $5 \mu \mathrm{g} / \mathrm{ml}$ acridine orange (Sigma-Aldrich; Merck, Tokyo, Japan) for $15 \mathrm{~min}$ at room temperature. Then, the samples were washed twice with $\mathrm{Ca}^{2+} / \mathrm{Mg}^{2+}$-free phosphate-buffered saline [PBS(-)] and immediately analyzed using a BD FACSCalibur flow cytometer (BD Biosciences, San Jose, CA, USA), or examined under a fluorescence microscope (BZ-8100; Keyence Corp., Tokyo, Japan). The experiments were performed three times. All numerical data were expressed as the mean \pm SD.

Apoptosis and cell death analysis. SW480 and HT-29 cells were prepared and treated as aforementioned, and the harvested cells were stained with a combination of Annexin V-fluorescein isothiocyanate (FITC) and propidium iodide (PI) (Annexin V-FITC apoptosis detection kit; BD Biosciences), according to the manufacturer's instructions. The samples were immediately analyzed by flow cytometer. This method allows the distinction between cells in early (Annexin V-FITC $/ \mathrm{PI}^{-}$) and late (Annexin $\mathrm{V}-\mathrm{FITC}^{+} / \mathrm{PI}^{+}$) apoptosis; however, in the present study, these subpopulations were counted together and expressed as a fraction of the apoptotic 
cells. Next, for the quantification of caspase-3/7 activity, cells were labeled with CellEvent caspase-3/7 green detection reagent (Life Technologies; Thermo Fisher Scientific, Inc.) and analyzed by flow cytometry. Alternatively, for calculating the proportion of dead cells, the cells were stained with trypan blue (Sigma-Aldrich; Merck KGaA), and dead cells (stained with trypan blue) and alive cells (unstained cells) were counted immediately. All experiments were performed three times, and all data were expressed as the mean \pm SD.

Statistical analysis. Statistical significance of the differences was determined using the unpaired, two-tailed Student's t-test for single comparisons and one-way ANOVA followed by Tukey's post hoc test for multiple comparisons. In addition, the interactions of radiation levels and different drug therapies were analyzed by two-way ANOVA. All analyses were performed using the JMP Pro 13.0 software (SAS Institute, Inc., Cary, NC, USA). Differences with $\mathrm{P}<0.05$ were considered statistically significant.

\section{Results}

IR activates the mTOR signaling pathway and induces autophagy in CRC cells. To investigate whether IR activates the mTOR signaling pathway and induces autophagy in human CRC cell lines, the levels of mTOR pathway-related proteins and autophagy-related proteins in SW480 and HT-29 cells before and after IR exposure were determined by western blotting (Fig. 1A and B). The levels of the phosphorylated isoforms of mTOR pathway-related proteins, S6 ribosomal protein and $4 \mathrm{E}-\mathrm{BP} 1$, normalized by the respective total proteins, were significantly increased after IR exposure in both cell lines, indicating that IR activated the mTOR signaling pathway in CRC cells. Similarly, conversion of LC3-I to LC3-II, which plays an important role in autophagosome formation, was observed, and the LC3-II/LC3-I ratios increased after IR exposure, whereas the levels of p62, which is consumed during autophagy, decreased after IR exposure, in both cell lines. These results indicate that IR induced autophagy in CRC cells. The time-dependent levels of p-S6 normalized by total S6 protein (p-S6/total S6 ratios) and LC3-II/LC3-I ratios in cells after the IR exposure are displayed in Fig. 1C and D. In both the cell lines, p-S6/total S6 ratios increased immediately after IR exposure and then decreased at $48 \mathrm{~h}$ after exposure, whereas LC3-II/LC3-I ratios increased slowly and remained high at $48 \mathrm{~h}$ after IR exposure. Similarly, the number of acidic autophagosomes stained with acridine orange significantly increased after IR exposure in a time-dependent manner, in both cell lines (Fig. 1E and F).

TEM and CQ combination therapy increases radiosensitivity in CRC cells. Next, whether mTOR inhibition and/or autophagy inhibition increases radiosensitivity in CRC cells was investigated, by studying the dose-dependent efficacies of IR in cells with or without TEM and/or CQ. In the clonogenic assay (Fig. 2A and B), a two-way ANOVA revealed the interaction of radiation levels and drug therapies in SW480 cells $(\mathrm{P}<0.001)$ and HT-29 cells $(\mathrm{P}<0.001)$. Although CQ or TEM monotherapy did not affect the suppressive effect of irradiation, the combination of TEM and CQ significantly enhanced the radiosensitivity at 2, 4 and 6 Gy in SW480 cells and at 4 and 6 Gy in HT-29 cells. In the MTS assay, TEM or CQ treatment in cells exposed to IR decreased cell proliferation, compared to that observed with IR exposure alone (Fig. 2C). Moreover, cell proliferation after the combination therapy of TEM and CQ was lower than that observed after the monotherapies with TEM or CQ.

TEM and CQ combination therapy inhibits the activation of mTOR pathway and autophagy in IR-exposed cells. Next, the levels of mTOR pathway-related proteins and autophagy-related proteins in each group were assessed (Fig. 3A and B). The levels of IR-induced phosphorylated isoforms of S6 and 4E-BP1 significantly decreased after TEM monotherapy but were not affected by CQ monotherapy. The IR-induced conversion of LC3-I to LC3-II significantly increased after CQ or TEM treatment and the LC3-II/LC3-I ratios were the highest after TEM and CQ combination therapy. Furthermore, p62 consumed by IR decreased after TEM monotherapy but increased after CQ monotherapy and was the highest after the combination therapy. Furthermore, acridine orange staining revealed that the number of IR-induced acidic autophagosomes increased after treatment with a combination of TEM and CQ, compared to that observed after monotherapy with TEM or CQ (Fig. 3B). These results were demonstrated in both SW480 and HT-29 cells.

TEM and CQ combination therapy induces apoptosis in IR-exposed cells. To analyze cell death in each group, apoptosis was analyzed via double staining with Annexin V-FITC and PI (Fig. 4A). The proportion of apoptotic cells, as Annexin V-positive cells, in each group are displayed in Fig. 4B. The apoptosis rate after IR exposure alone (5.5\%) was significantly higher than that of the control (3.1\%), which indicated that IR induced apoptosis in SW480 cells. TEM or CQ treatment with IR exposure in SW480 cells increased the apoptotic rate to 6.6 and $7.4 \%$, respectively, but these differences were not significant; however, treatment with a combination of TEM and CQ with IR exposure significantly increased the apoptotic rate up to $14.3 \%$, compared to that observed after IR exposure with or without TEM or CQ single agents. Similarly, the proportion of dead cells stained with trypan blue in SW480 cells treated with IR and a combination of TEM and CQ (5.2\%) were significantly higher than those in the control cells $(1.1 \%)$ and cells treated with IR alone (2.5\%), TEM alone (2.9\%), and CQ alone (3.3\%; Fig. 4D). The proportion of activated caspase-3/7 in SW480 cells treated with IR and a combination of TEM and CQ (15.5\%) were significantly higher than those in control cells (3.5\%) and cells treated with IR alone (7.0\%), TEM alone (9.3\%), and CQ alone (11.0\%; Fig. 4C). Furthermore, similar results were obtained from HT-29 cells.

\section{Discussion}

The PI3K/AKT/mTOR pathway is an intracellular signaling pathway involving the phosphorylation of proteins downstream of tyrosine kinase receptors, resulting in phosphorylation of ribosomal protein S6 and 4E-BP1 (14). In cancer cells, the activation of mTOR signaling promotes tumor growth and 
A

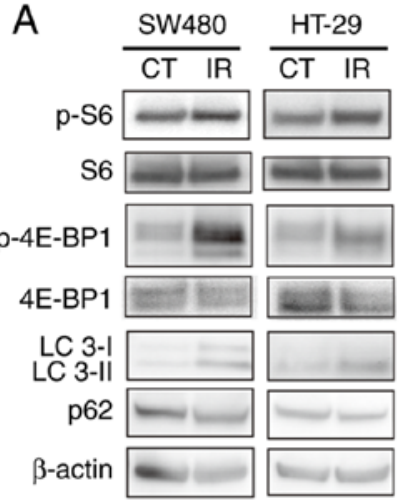

$\mathrm{B}$

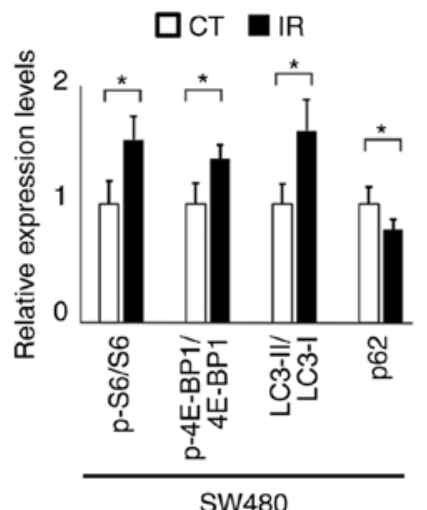

$\square C T=I R$

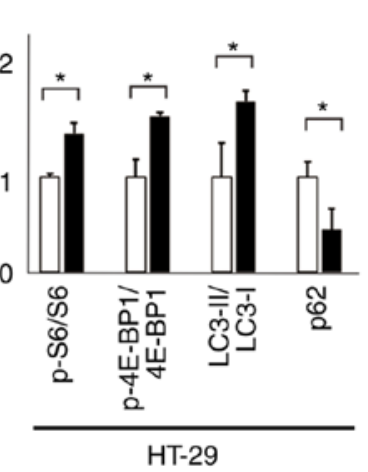

C
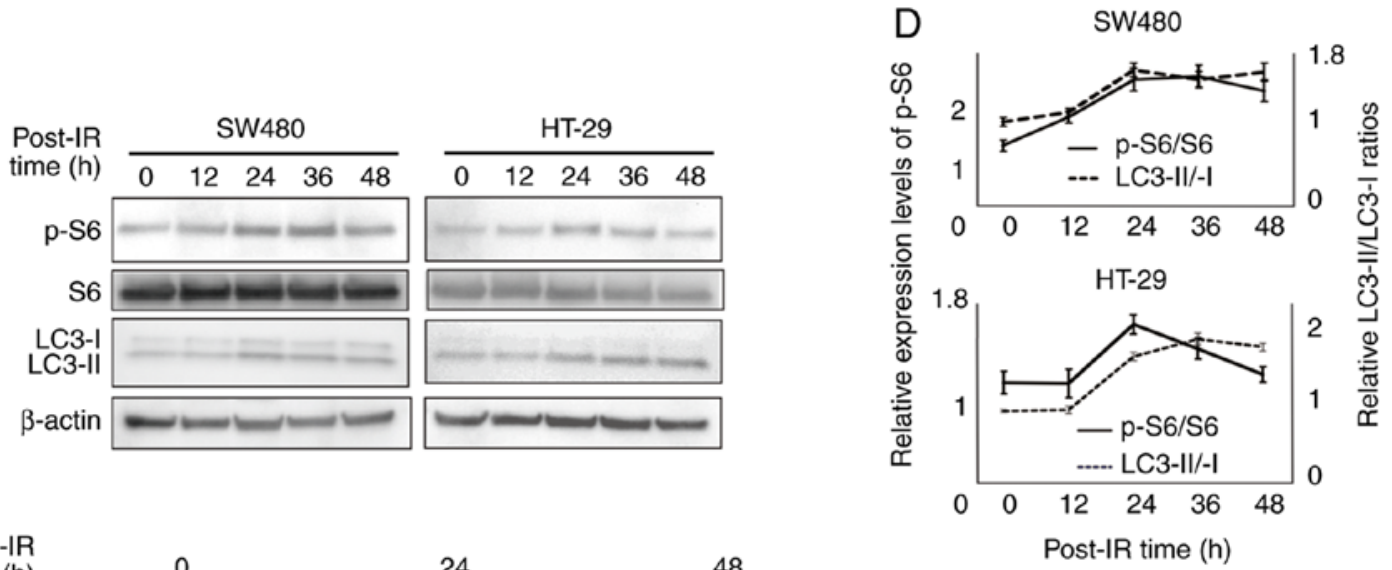

E Post-IR time (h) 0
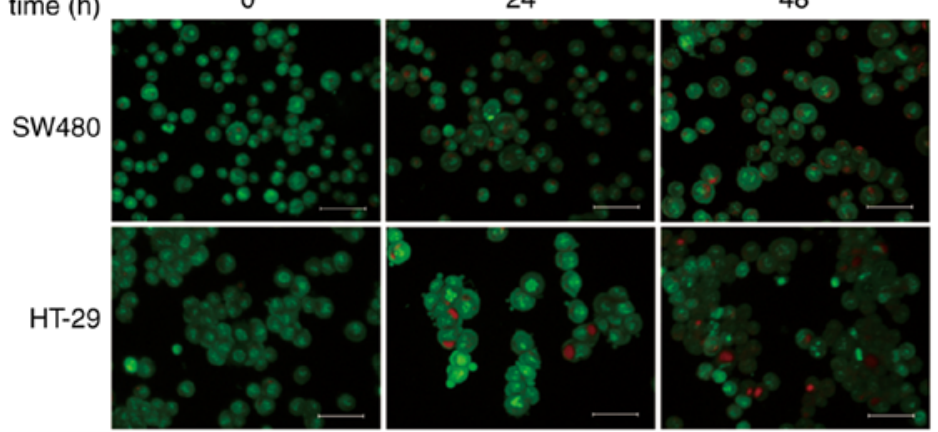

F

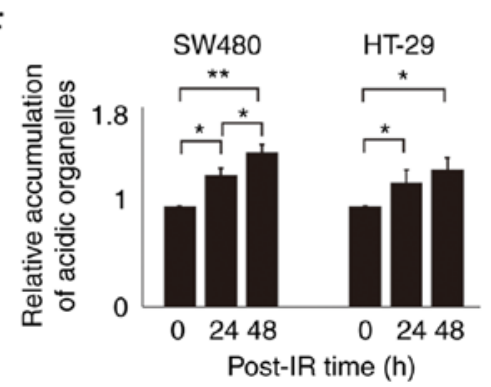

Figure 1. Ionizing radiation activates the mTOR pathway and induces autophagy in human colorectal cancer cells. HT-29 and SW480 cells were treated with ionizing radiation at a dose of $4 \mathrm{~Gy}$ and mTOR pathway activation and autophagy induction were investigated. (A) Western blot analysis of the mTOR pathway-related proteins, p-S6, total S6, p-4E-BP1, and total 4E-BP1; and autophagy-related proteins LC3B-II/I and SQSTM1/p62 in cell lines $24 \mathrm{~h}$ after radiotherapy. $\beta$-actin was used as the control. (B) Bar plots represent the relative expression levels of each protein, as determined via densitometric analysis. The mTOR pathway-related proteins were presented as the phosphorylated isoform ratios normalized by the respective total proteins. The autophagy-related proteins were presented as LC3-II/LC3-I ratios and the p62 expression levels were normalized to $\beta$-actin levels. The data are expressed as the mean \pm SD from the results of three independent experiments $(n=3)$. ${ }^{*} \mathrm{P}<0.05$. Unpaired Student's t-tests were used. (C) $\mathrm{p}-\mathrm{S} 6$, total S6, and LC3B-II/I levels at different intervals after radiotherapy. (D) Line graphs represent the changes in the expression levels of p-S6/total S6 and LC3-II/LC3-I ratio, as determined via densitometric analysis. The data are expressed as the mean $\pm \mathrm{SD}(\mathrm{n}=3)$. (E) Acridine orange staining and fluorescence microscopy results for the accumulation of acidic vesicular organelles (bright red fluorescence) in cell lines $24 \mathrm{~h}$ after radiotherapy. Scale bar, $20 \mu \mathrm{m}$. (F) Bar plots represent flow cytometric results for formation of acidic vesicular organelles in cell lines $24 \mathrm{~h}$ after radiotherapy. The values of each therapy group were standardized by the value of the control, without any treatment. The data are expressed as the mean $\pm \mathrm{SD}(\mathrm{n}=3) .{ }^{*} \mathrm{P}<0.05,{ }^{* *} \mathrm{P}<0.01$. One-way ANOVA was used. p-S6, phospho-S6 ribosomal protein; p-4E-BP1, phospho-eukaryotic translation initiation factor 4E-binding protein 1; SQSTM1, sequestosome-1.

angiogenesis and inhibits apoptosis $(14,15)$. Furthermore, the PI3K/AKT/mTOR pathway and autophagy are known to play important roles in cancer radioresistance. In the present study, the effect of TEM (mTOR inhibitor) and CQ (autophagy inhibitor) combination therapy was investigated on the radiosensitivity of CRC cells.

In the present study, S6 and 4E-BP1 were phosphorylated after IR exposure with a peak at 24-36 h after IR exposure, indicating that mTOR signaling was activated by IR. The activation of mTOR signaling by IR has been reported in endometrial and breast cancer cells as well as human umbilical vein endothelial cells $(3,16,17)$ but not in CRC cells . Since mTOR inhibitor has been reported to inhibit the repair of IR-induced DNA damage in breast cancer cells, mTOR signaling activated by IR is considered to play an important role in radioresistance (4).

Generally during the autophagic process, the autophagy substrate SQSTM1/p62 is consumed, LC3 is converted from 

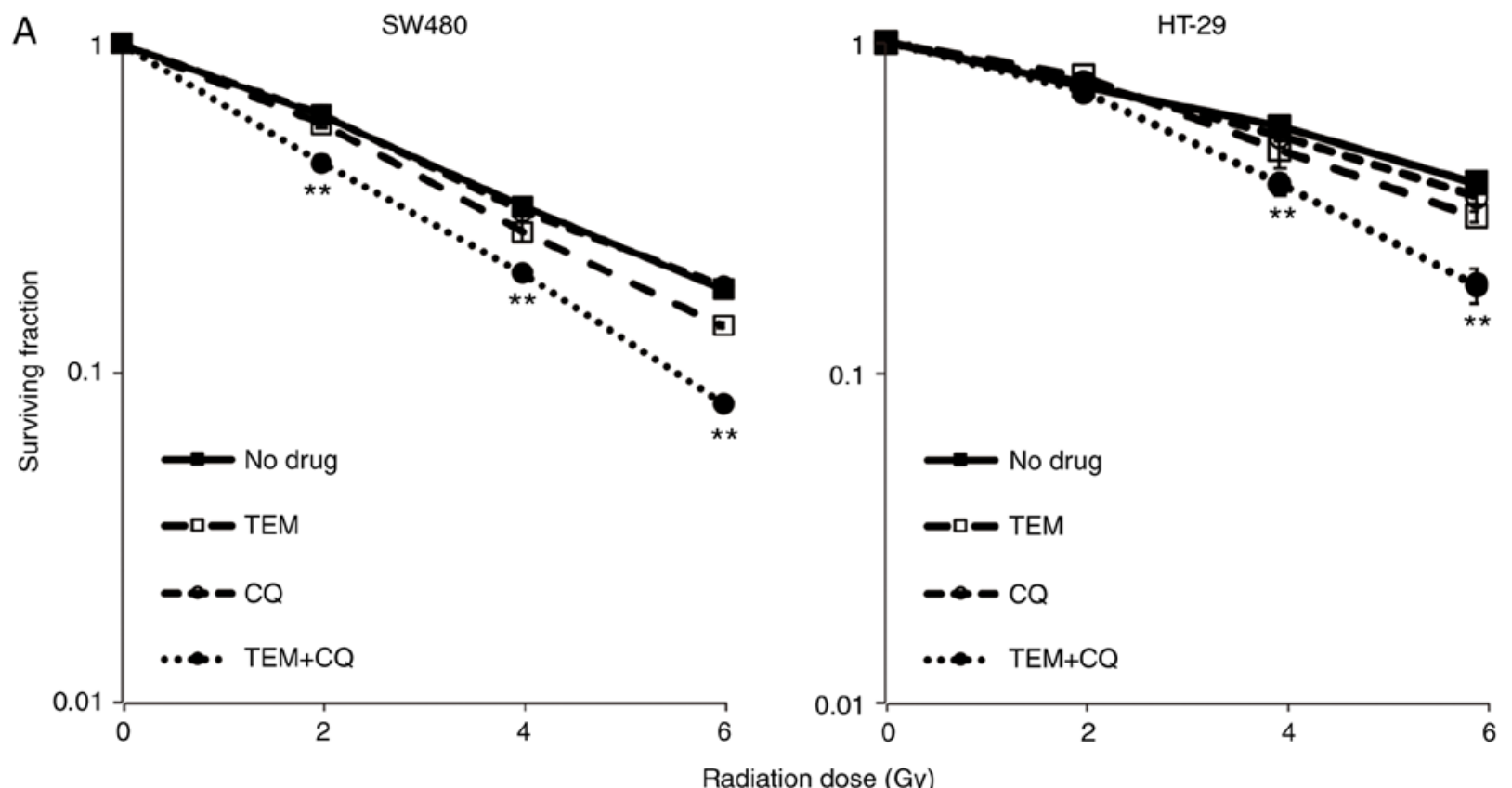

B
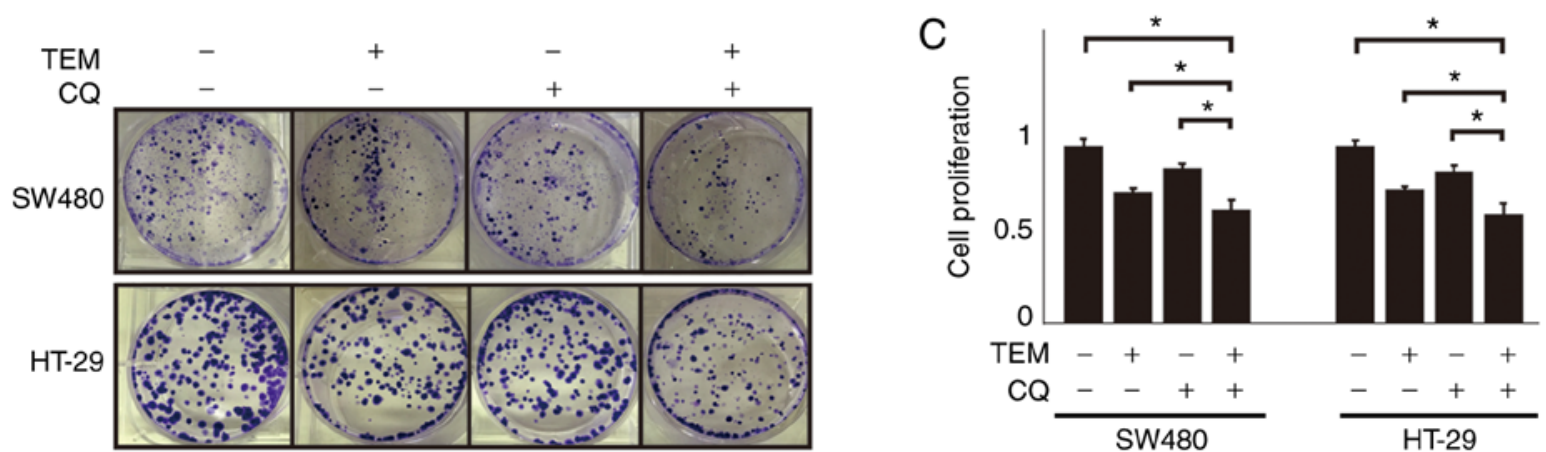

Figure 2. Combination of TEM and CQ increases the anti-proliferative effect of IR in HT-29 and SW480 cells. Cells were treated with $80 \mathrm{nM}$ TEM and/or $20 \mu \mathrm{M}$ CQ followed by several doses of IR. (A) Clonogenic assay results revealing the colony-forming ability of HT-29 and SW480 cells in the presence of different doses of IR with or without TEM and/or CQ. Based on the results of a two-way ANOVA, only the drug groups that significantly influenced the effect of IR at the individual radiation doses have been indicated. The data are expressed as the mean $\pm \mathrm{SD}(\mathrm{n}=3) ;{ }^{* *} \mathrm{P}<0.01$. Cells treated with combination of TEM and CQ vs. untreated cells at the individual IR doses. (B) Results of crystal violet staining revealing surviving cell colonies on $10 \mathrm{~cm}$ dishes. (C) Results of the MTS assay revealing proliferation of cells 3 days after 4 Gy radiotherapy with or without TEM and/or CQ. The values in each group were standardized by the value of the control without radiotherapy. The data are expressed as the mean $\pm \mathrm{SD}(\mathrm{n}=3)$. ${ }^{*} \mathrm{P}<0.05$. One-way ANOVA was used. TEM, temsirolimus; $\mathrm{CQ}$, chloroquine; IR, ionizing radiation.

its isotype I to II, and acidic organelles are accumulated in the cell $(18,19)$. In particular, the LC3-II/LC3-I ratio is considered an important parameter to evaluate the degree of autophagy. In the present study, p62 levels decreased and the LC3-II/LC3-I ratios and accumulation of acidic organelles increased with a peak at $36 \mathrm{~h}$ after IR exposure, indicating that autophagy was induced by IR. These results are consistent with previous studies on IR-induced autophagy in many solid cancer cells, including CRC cells, indicating that IR-induced autophagy may play a cytoprotective role (10-12).

Several studies have reported the irradiation-induced activation of the mTOR pathway $(3,16,17)$ and autophagy pathway (10-12) in different cell lines, despite the suppressive effect of the activated mTOR pathway on autophagy. It has been reported that autophagy can be induced via several pathways after radiation, such as those involving accumulation of reactive oxygen (ROS), calcium ions and mitochondrial damage (7). The present results indicated that irradiation induced autophagy activation independently of the mTOR pathway and that this activation overcame the suppressive effect of the mTOR pathway; however, further studies are warranted to confirm this. TEM treatment in combination with irradiation increased the LC3-II/LC3-I ratio and p62 consumption (Fig. 3), indicating the suppressive effect of the mTOR pathway on autophagy and enhancement of autophagy by mTOR inhibition.

Next, a clonogenic assay was performed using TEM and/or CQ, to determine whether the inhibition of mTOR signaling and/or autophagy increases radiosensitivity in CRC cells. In both SW480 and HT-29 cells, neither TEM nor CQ monotherapy influenced the effect of radiation, but the interaction of the combination of TEM and CQ and radiation levels was demonstrated by two-way ANOVA. Western blotting revealed that TEM alone inhibited IR-induced mTOR signaling but induced autophagy, as indicated by decreased p62 and increased LC3-II/LC3-I ratios. mTOR is known to be a strong factor for inhibiting autophagy; mTOR C1 phosphorylates ULK1 and suppresses its function, which 
A

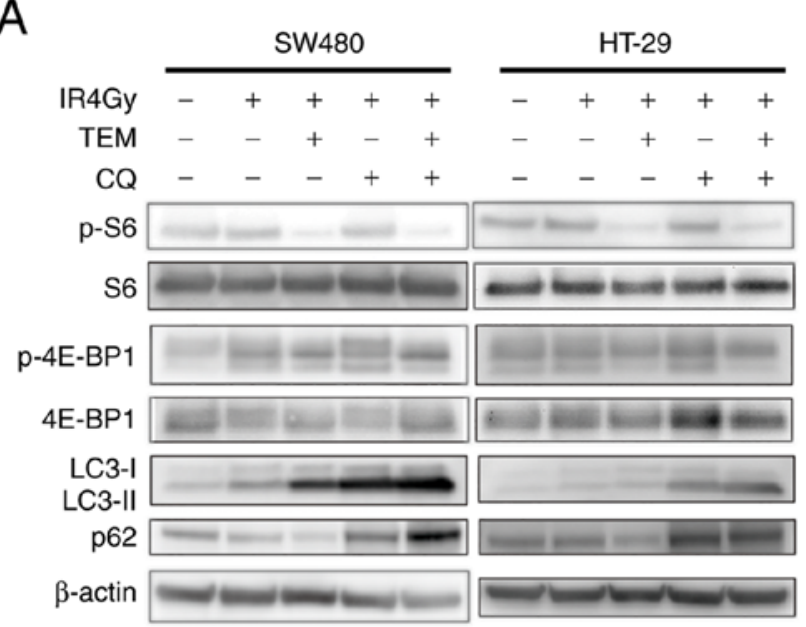

C

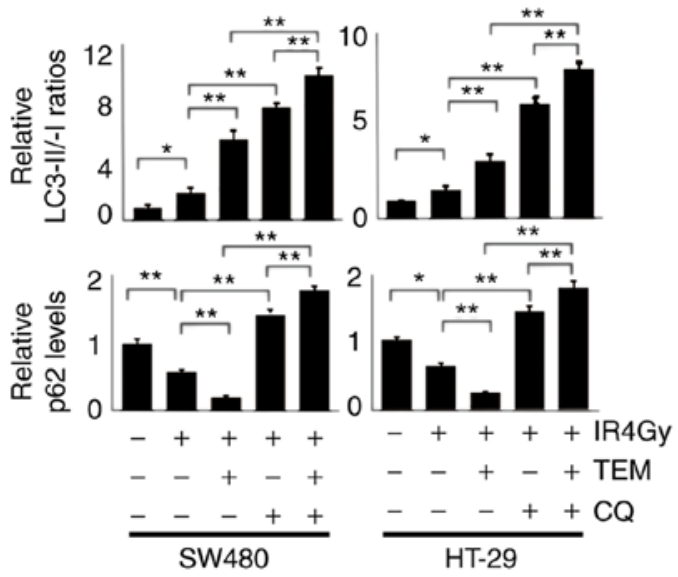

B

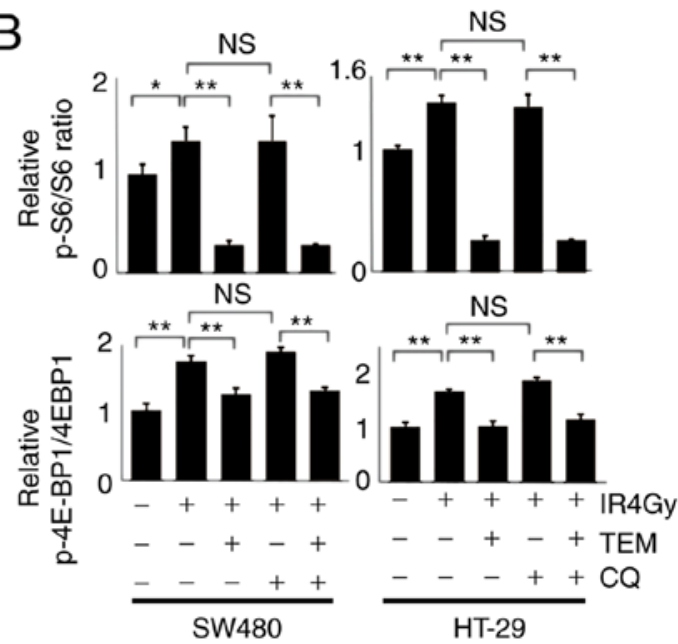

D

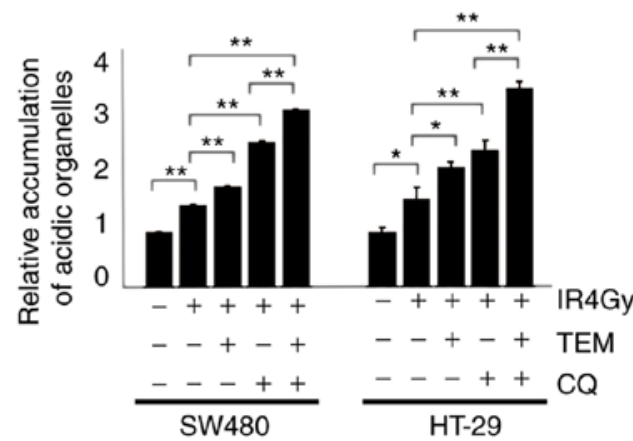

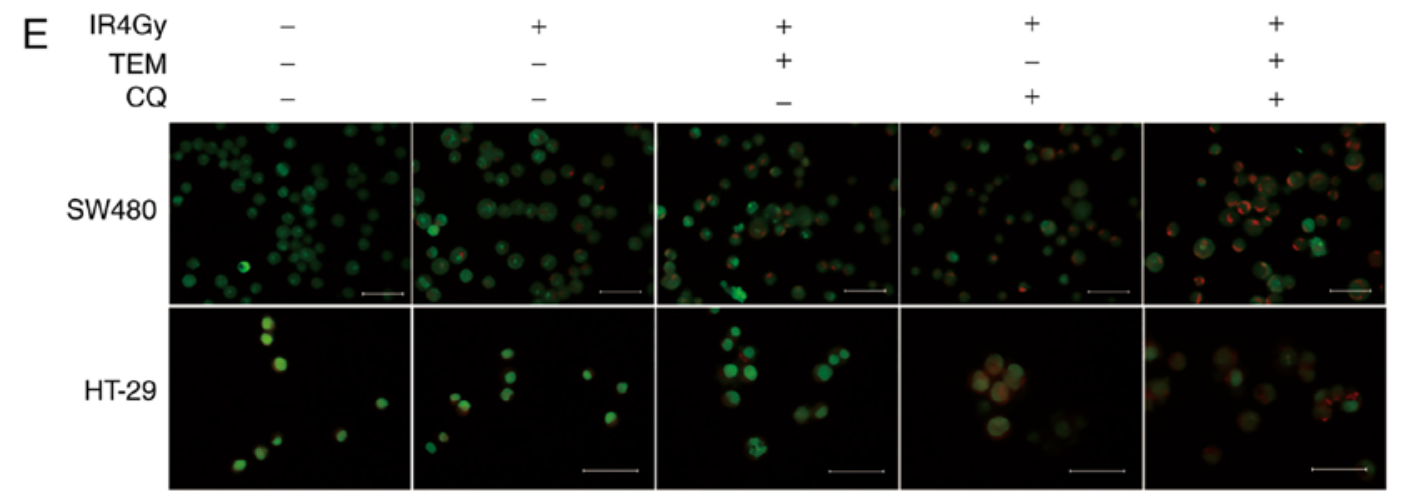

Figure 3. Combination of TEM, CQ and IR downregulates mTOR pathway-related proteins and autophagy-related proteins in SW480 and HT-29 cells. The cells were treated with $80 \mathrm{nM}$ TEM and/or $20 \mu \mathrm{M} \mathrm{CQ}$ followed by $4 \mathrm{~Gy}$ radiotherapy and examined $24 \mathrm{~h}$ after radiotherapy. (A) Western blot analysis of mTOR pathway- and autophagy-related proteins. (B) Bar plots represent the relative expression levels of mTOR-related proteins, p-S6/total S6, and p-4E-BP1/total 4E-BP1, as determined via densitometric analysis. The data are expressed as the mean $\pm \mathrm{SD}(\mathrm{n}=3)$. ${ }^{*} \mathrm{P}<0.05,{ }^{* *} \mathrm{P}<0.01$. One-way ANOVA was used. (C) Bar plots represent the relative expression levels of autophagy-related proteins, LC3-II/LC3-I ratios, and p62 levels. The data are expressed as the mean \pm SD $(\mathrm{n}=3) .{ }^{*} \mathrm{P}<0.05,{ }^{* *} \mathrm{P}<0.01$. One-way ANOVA was used. (D) Bar plots represent flow cytometric results revealing the formation of acridine orange-stained acidic vesicular organelles in cells $24 \mathrm{~h}$ after radiotherapy. The values of each group were standardized by the value of the control without any treatment. The data are expressed as the mean $\pm \mathrm{SD}(\mathrm{n}=3)$. ${ }^{*} \mathrm{P}<0.05,{ }^{* *} \mathrm{P}<0.01$. One-way ANOVA was used. (E) Fluorescence microscopy images showing acridine orange-stained autophagic vesicles in cells. Scale bar, $20 \mu \mathrm{m}$. NS, not significant; TEM, temsirolimus; CQ, chloroquine; IR, ionizing radiation; p-S6, phospho-S6 ribosomal protein; p-4E-BP1, phospho-eukaryotic translation initiation factor 4E-binding protein 1.

in turn suppresses the formation of autophagosomes (7). An mTOR inhibitor has been reported to induce autophagy by dephosphorylating ULK1 and promoting the formation of autophagosomes. In contrast, CQ alone was revealed to increase the level of $\mathrm{p} 62$, indicating autophagy inhibition since CQ inhibits autophagy in its late phase $(9,20)$. As anticipated, CQ monotherapy did not affect the levels of pS6 and p4E-BP1, suggesting that CQ does not influence mTOR signaling. Cells treated with a combination of TEM and CQ revealed the highest levels of p62 and LC3-II/LC3-I ratios, confirming the inhibition of IR-activated mTOR signaling by TEM and the late phase inhibition of TEM-induced autophagy 


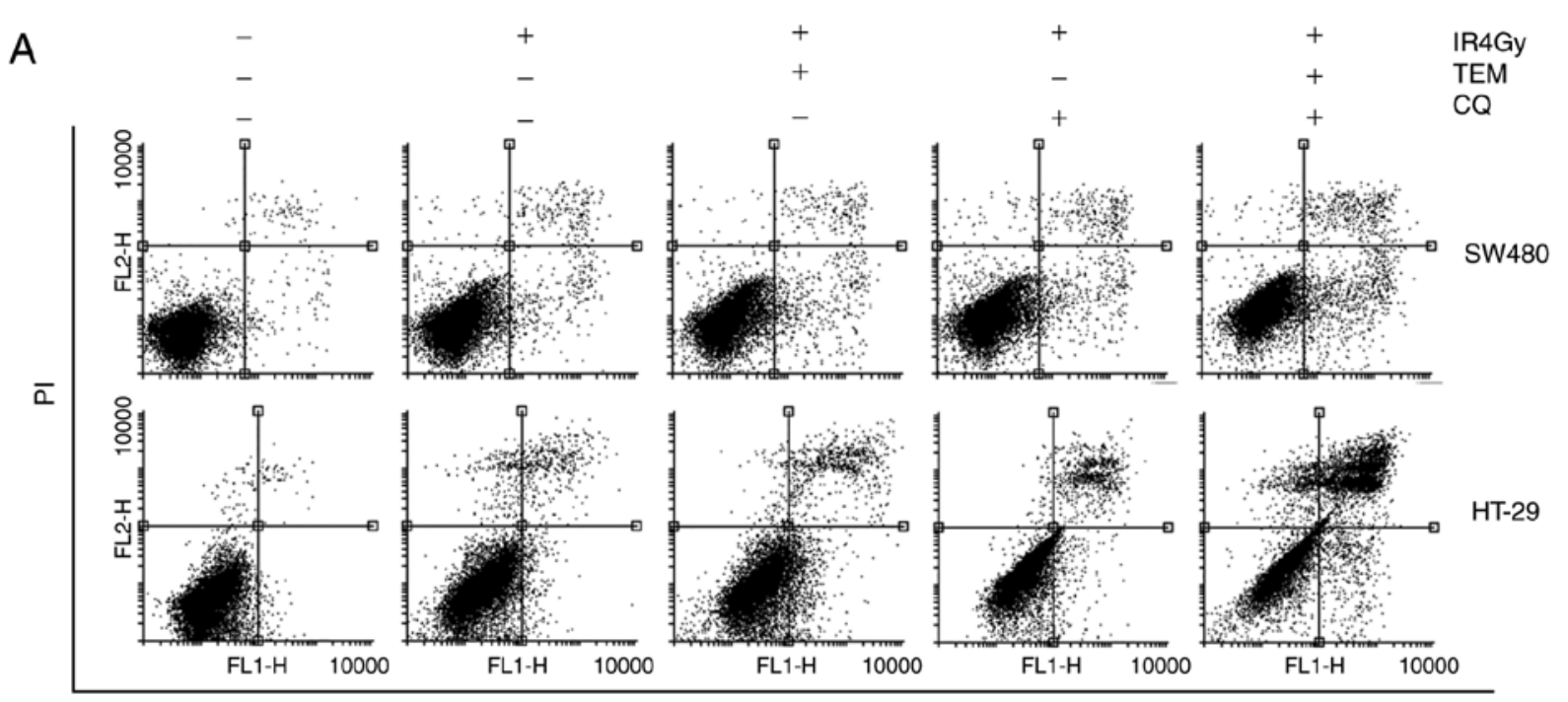

Annexin V+FITC
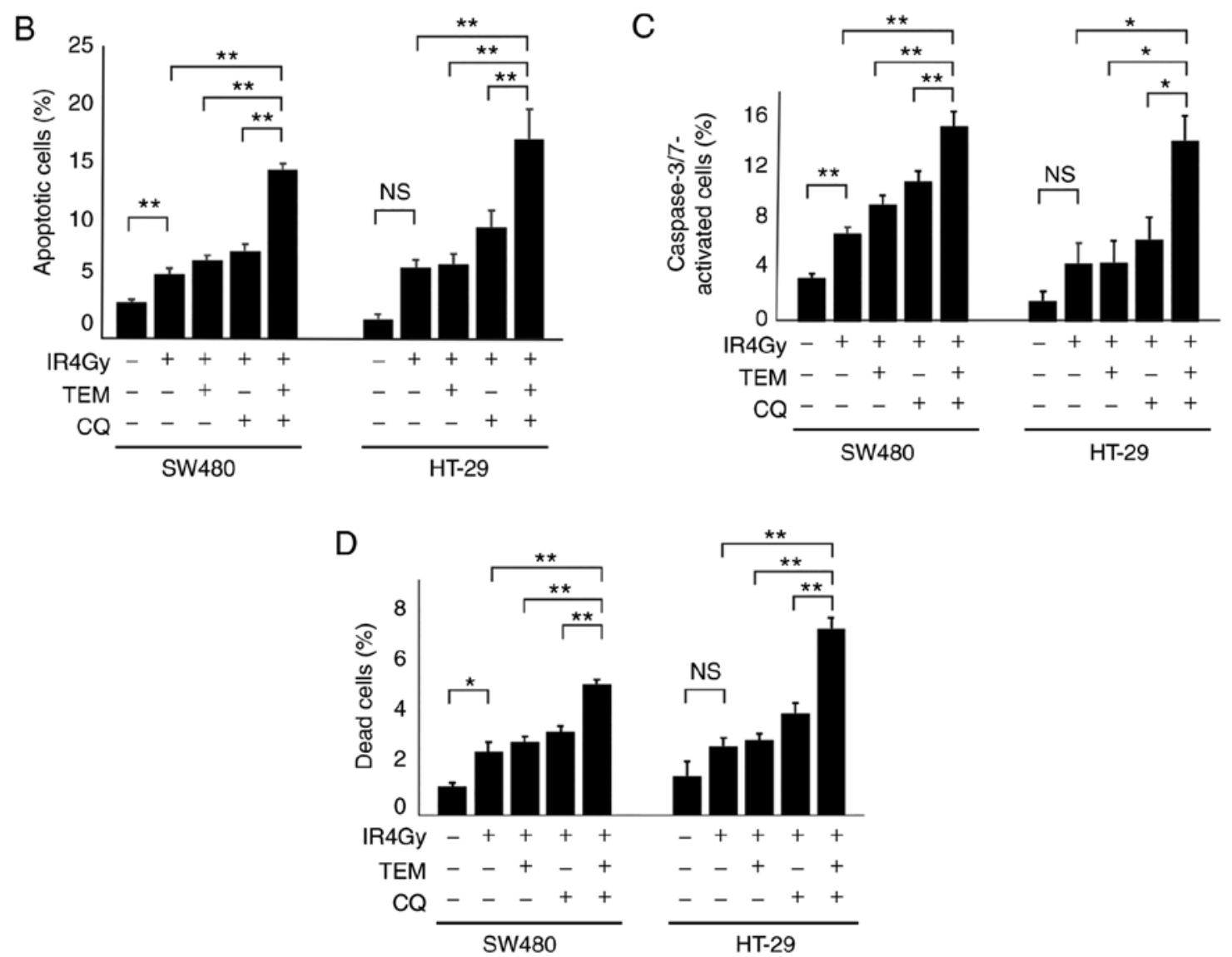

Figure 4. Combination of TEM, CQ and IR induces apoptosis in SW480 and HT-29 cells. The cells were treated with $80 \mathrm{nM}$ TEM and/or $20 \mu \mathrm{M}$ CQ followed by $4 \mathrm{~Gy}$ radiotherapy and examined $48 \mathrm{~h}$ after radiotherapy. (A) FACS analysis of apoptosis based on Annexin $\mathrm{V}$ and propidium iodide (PI) staining in different treatment groups. The cells in the upper-right and lower-right quadrants represent apoptotic cells. The data are expressed as the mean \pm SD ( $n=3$ ). One-way ANOVA was used. (B) Bar graph revealing the summed relative number of apoptotic cells in the different treatment groups. The data are expressed as the mean $\pm \mathrm{SD}(\mathrm{n}=3) .{ }^{* *} \mathrm{P}<0.01$. One-way ANOVA was used. (C) Percentage of cells with activated caspase-3/7, as detected using FACS analysis. ${ }^{*} \mathrm{P}<0.05,{ }^{* *} \mathrm{P}<0.01$. (D) Trypan blue staining revealed the percentage of dead cells in different treatment groups. The data are expressed as the mean $\pm \mathrm{SD}(\mathrm{n}=3)$. ${ }^{*} \mathrm{P}<0.05,{ }^{* * *} \mathrm{P}<0.01$. One-way ANOVA was used. NS, not significant; TEM, temsirolimus; CQ, chloroquine; IR, ionizing radiation.

by CQ. Therefore, autophagy induction after TEM treatment and active mTOR signaling after CQ treatment indicate that TEM or CQ alone does not affect the radiosensitivity of cells and that both inhibition of mTOR and autophagy collectively enhance radiosensitivity. Although the radiosensitizing effect of the mTOR inhibitor alone was reported in pancreatic, breast, and prostate cancer cells via in vivo and in vitro studies $(3,21,22)$, the radiosensitizing effect of rapamycin was not observed in a clinical trial of rectal cancer (5). Similarly, the radiosensitizing effect of autophagy inhibitor 3-MA was reported in breast cancer cells (23), but CQ did not affect radiosensitivity in CRC cells (24). 
According to these studies, the radiosensitizing effects of an mTOR inhibitor alone or CQ alone differed among the cell lines (25). In contrast, the combination of an mTOR inhibitor and CQ was reported to improve the anti-proliferative effect in several cells, including CRC cells $(26,27)$, however, the radiosensitizing effect of this combination has not yet been reported. Hence, it was considered that inhibition of mTOR signaling or autophagy alone was insufficient for enhancing radiosensitivity, but co-inhibition of both these processes is essential in CRC cells.

Cell death analysis revealed a significantly high apoptosis rate after TEM and CQ combination therapy but not after TEM or CQ monotherapy. Previous studies reported the preservation of phosphorylated AKT, an anti-apoptotic protein after treatment with an mTOR inhibitor, indicating that it does not induce apoptosis (28). Autophagy maintains the quality of mitochondria, thus preventing the accumulation of intracellular ROS (7). Although autophagic inhibition has been reported to induce apoptosis through aberrant ROS accumulation, in the present study no significant increase was revealed in the apoptosis rates after treatment with $\mathrm{CQ}$ alone. In contrast, the combination of an mTOR inhibitor and CQ was reported induce apoptosis in a synergistic manner, consistent with the results obtained in our study $(8,9,27)$. An mTOR inhibitor induces autophagosome formation and CQ leads to the permeabilization of the autophagosome membrane, resulting in the induction of apoptosis (29-31). In the present study, the accumulation of autophagosomes was observed in IR-exposed cells, but the accumulation was enhanced in cells treated with IR and TEM. Thus, it was hypothesized that adding CQ in the presence of excessive autophagosome accumulation may contribute to increased apoptosis via autophagosome membrane permeabilization.

There are several limitations of the present study. First, this is an in vitro study, and the findings from an in vivo study may not be consistent with our findings. Furthermore, although the safety of the combination of TEM and CQ has been demonstrated in a phase-I clinical study for several solid cancers (32), clinical studies investigating the effect of this combination on radiosensitivity have not yet been performed, and prospective clinical trials are warranted to confirm our results. Second, the radiosensitizing effect of the combination of TEM and CQ in this study may be attributed to their unique effects, and a combination of other mTOR and autophagy inhibitors may not yield results consistent with those obtained in the present study. Therefore, future investigations using various mTOR and autophagy inhibitors are required. This study would be greatly strengthened by future validating studies using clinical colorectal cancer tissues or colorectal cancer xenograft in vivo models, which were beyond the scope of the present study.

In conclusion, the radiosensitizing effect of the combination of TEM and CQ was demonstrated in CRC cells. This combination therapy may be considered as an effective chemotherapy combined with radiotherapy in patients undergoing neoadjuvant CRT for rectal cancer.

\section{Acknowledgements}

Not applicable.

\section{Funding}

The present study was supported by Grants-in-Aid for Scientific Research (grant nos. 16K07143, 16K07161, 17K10620, 17K10621, 17K10623 and 18K07194) from the Japan Society for the Promotion of Science. This research was also supported by the Project for Cancer Research and Therapeutic Evolution (P-CREATE; grant no. 18cm0106502h0003) from the Japan Agency for Medical Research and Development (AMED).

\section{Availability of data and materials}

All datasets generated during this study are included in this published article.

\section{Authors' contributions}

HSh, KK, KH and HN aided in the concept and design of the project. HSh, TT, TN, KO, KS, MK, KM, SE and HSo contributed to manuscript preparation and data collection. HSh and $\mathrm{KK}$ interpreted the results, analyzed the data, and revised the manuscript. All authors read and approved the manuscript and agree to be accountable for all aspects of the research in ensuring that the accuracy or integrity of any part of the work are appropriately investigated and resolved.

\section{Ethics approval and consent to participate}

Not applicable.

\section{Patient consent for publication}

Not applicable.

\section{Competing interests}

The authors declare that they have no competing interests.

\section{References}

1. Martin ST, Heneghan HM and Winter DC: Systematic review and meta-analysis of outcomes following pathological complete response to neoadjuvant chemoradiotherapy for rectal cancer. $\mathrm{Br}$ J Surg 99: 918-928, 2012.

2. Rödel C, Liersch T, Becker H, Fietkau R, Hohenberger W, Hothorn T, Graeven U, Arnold D, Lang-Welzenbach M, Raab HR, et al: Preoperative chemoradiotherapy and postoperative chemotherapy with fluorouracil and oxaliplatin versus fluorouracil alone in locally advanced rectal cancer: Initial results of the German CAO/ARO/AIO-04 randomised phase 3 trial trial. Lancet Oncol 13: 679-687, 2012.

3. Albert JM, Kim KW, Cao C and Lu B: Targeting the Akt/mammalian target of rapamycin pathway for radiosensitization of breast cancer. Mol Cancer Ther 5: 1183-1189, 2006.

4. Chen H, Ma Z, Vanderwaal RP, Feng Z, Gonzalez-Suarez I, Wang S, Zhang J, Roti Roti JL, Gonzalo S and Zhang J: The mTOR inhibitor rapamycin suppresses DNA double-strand break repair. Radiat Res 175: 214-224, 2011.

5. Buijsen J, van den Bogaard J, Jutten B, Belgers E, Sosef M, Leijtens JW, Beets GL, Jansen RL, Riedl RG, Clarijs R, et al: A phase I-II study on the combination of rapamycin and short course radiotherapy in rectal cancer. Radiother Oncol 116: 214-220, 2015.

6. Chaurasia M, Bhatt AN, Das A, Dwarakanath BS and Sharma K: Radiation-induced autophagy: Mechanisms and consequences. Free Radic Res 50: 273-290, 2016. 
7. Hu L, Wang H, Huang L, Zhao Y and Wang J: Crosstalk between autophagy and intracellular radiation response (Review). Int J Oncol 49: 2217-2226, 2016.

8. Rosich L, Xargay-Torrent S, López-Guerra M, Campo E, Colomer D and Roué G: Counteracting autophagy overcomes resistance to everolimus in mantle cell lymphoma. Clin Cancer Res 18: 5278-5289, 2012.

9. Kaneko M, Nozawa H, Hiyoshi M, Tada N, Murono K, Nirei T, Emoto S, Kishikawa J, Iida Y, Sunami E, et al: Temsirolimus and chloroquine cooperatively exhibit a potent antitumor effect against colorectal cancer cells. J Cancer Res Clin Onco 140: 769-781, 2014.

10. Mo N, Lu YK, Xie WM, Liu Y, Zhou WX, Wang HX, Nong L, Jia YX, Tan AH, Chen Y, et al: Inhibition of autophagy enhances the radiosensitivity of nasopharyngeal carcinoma by reducing Rad51 expression. Oncol Rep 32: 1905-1912, 2014.

11. Sun Q, Liu T, Yuan Y, Guo Z, Xie G, Du S, Lin X, Xu Z, Liu M, Wang W, et al: MiR-200c inhibits autophagy and enhances radiosensitivity in breast cancer cells by targeting UBQLN1. Int J Cancer 136: 1003-1012, 2015.

12. Apel A, Herr I, Schwarz H, Rodemann HP and Mayer A: Blocked autophagy sensitizes resistant carcinoma cells to radiation therapy. Cancer Res 68: 1485-1494, 2008.

13. Okuno T, Kawai K, Hata K, Murono K, Emoto S, Kaneko M, Sasaki K, Nishikawa T, Tanaka T and Nozawa H: SN-38 Acts as a radiosensitizer for colorectal cancer by inhibiting the radiation-induced Up-regulation of HIF-1 $\alpha$. Anticancer Res 38 : 3323-3331, 2018.

14. Saxton RA and Sabatini DM: mTOR signaling in growth, metabolism, and disease. Cell 168: 960-976, 2017.

15. Fingar DC, Richardson CJ, Tee AR, Cheatham L, Tsou C and Blenis J: mTOR controls cell cycle progression through its cell growth effectors S6K1 and 4E-BP1/eukaryotic translation initiation factor 4E. Mol Cell Biol 24: 200-216, 2004.

16. Miyasaka A, Oda K, Ikeda Y, Sone K, Fukuda T, Inaba K, Makii C, Enomoto A, Hosoya N, Tanikawa M, et al: PI3K/mTOR pathway inhibition overcomes radioresistance via suppression of the HIF1- $\alpha$ /VEGF pathway in endometrial cancer. Gynecol Oncol 138: 174-180, 2015

17. Edwards E, Geng L, Tan J, Onishko H, Donnelly E and Hallahan DE: Phosphatidylinositol 3-kinase/Akt signaling in the response of vascular endothelium to ionizing radiation. Cancer Res 62: 4671-4677, 2002.

18. Nishikawa T, Tsuno NH, Okaji Y, Shuno Y, Sasaki K, Hongo K, Sunami E, Kitayama J, Takahashi K and Nagawa H: Inhibition of autophagy potentiates sulforaphane-induced apoptosis in human colon cancer cells. Ann Surg Oncol 17: 592-602, 2010.

19. Sasaki K, Tsuno NH, Sunami E, Tsurita G, Kawai K, Okaji Y, Nishikawa T, Shuno Y, Hongo K, Hiyoshi M, et al: Chloroquine potentiates the anti-cancer effect of 5-fluorouracil on colon cancer cells. BMC Cancer 10: 370, 2010.

20. Al-Bari MA: Chloroquine analogues in drug discovery: New directions of uses, mechanisms of actions and toxic manifestations from malaria to multifarious diseases. J Antimicrob Chemother 70: 1608-1621, 2015.
21. Cao C, Subhawong T, Albert JM, Kim KW, Geng L, Sekhar KR, Gi YJ and Lu B: Inhibition of mammalian target of rapamycin or apoptotic pathway induces autophagy and radiosensitizes PTEN nulll prostate cancer cells. Cancer Res 66: 10040-10047, 2006.

22. Manegold PC, Paringer C, Kulka U, Krimmel K, Eichhorn ME, Wilkowski R, Jauch KW, Guba M and Bruns CJ: Antiangiogenic therapy with mammalian target of rapamycin inhibitor RAD001 (Everolimus) increases radiosensitivity in solid cancer. Clin Cancer Res 14: 892-900, 2008.

23. Lomonaco SL, Finniss S, Xiang C, Decarvalho A, Umansky F, Kalkanis SN, Mikkelsen T and Brodie C: The induction of autophagy by gamma-radiation contributes to the radioresistance of glioma stem cells. Int J Cancer 125: 717-722, 2009.

24. Yan C, Luo L, Goto S, Urata Y, Guo CY, Doi H, Kitazato K and Li TS: Enhanced autophagy in colorectal cancer stem cells does not contribute to radio-resistance. Oncotarget 7: 45112-45121, 2016.

25. Bristol ML, Emery SM, Maycotte P, Thorburn A, Chakradeo S and Gewirtz DA: Autophagy inhibition for chemosensitization and radiosensitization in cancer: Do the preclinical data support this therapeutic strategy? J Pharmacol Exp Ther 344: 544-552, 2013.

26. Enzenmuller S, Gonzalez P, Debatin KM and Fulda S: Chloroquine overcomes resistance of lung carcinoma cells to the dual PI3K/mTOR inhibitor PI103 by lysosome-mediated apoptosis. Anticancer Drugs 24: 14-19, 2013.

27. Seitz C, Hugle M, Cristofanon S, Tchoghandjian A and Fulda S: The dual PI3K/mTOR inhibitor NVP-BEZ235 and chloroquine synergize to trigger apoptosis via mitochondrial-lysosomal cross-talk. Int J Cancer 132: 2682-2693, 2013.

28. Choo AY, Yoon SO, Kim SG, Roux PP and Blenis J: Rapamycin differentially inhibits S6Ks and 4E-BP1 to mediate cell-type-specific repression of mRNA translation. Proc Natl Acad Sci USA 105: 17414-17419, 2008.

29. Shi TT, Yu XX, Yan LJ and Xiao HT: Research progress of hydroxychloroquine and autophagy inhibitors on cancer. Cancer Chemother Pharmacol 79: 287-294, 2017.

30. Boya P, Gonzalez-Polo RA, Poncet D, Andreau K, Vieira HL, Roumier T,Perfettini JL and Kroemer G: Mitochondrial membrane permeabilization is a critical step of lysosome-initiated apoptosis induced by hydroxychloroquine. Oncogene 22: 3927-3936, 2003.

31. Lakhter AJ, Sahu RP, Sun Y, Kaufmann WK, Androphy EJ, Travers JB and Naidu SR: Chloroquine promotes apoptosis in melanoma cells by inhibiting $\mathrm{BH} 3$ domain-mediated PUMA degradation. J Invest Dermatol 133: 2247-2254, 2013.

32. Rangwala R, Chang YC, Hu J, Algazy KM, Evans TL, Fecher LA, Schuchter LM, Torigian DA, Panosian JT, Troxel AB, et al: Combined MTOR and autophagy inhibition: Phase I trial of hydroxychloroquine and temsirolimus in patients with advanced solid tumors and melanoma. Autophagy 10: 1391-1402, 2014. 